\title{
Gravitational Waves of Jet Precession in Gamma-ray Bursts
}

\author{
Mou-Yuan Sun ${ }^{1}$, Tong $\mathrm{Liu}^{1}$, Wei-Min $\mathrm{Gu}^{1,2}$, and $\mathrm{Ju}-\mathrm{Fu} \mathrm{Lu}^{1}$ \\ tongliu@xmu.edu.cn
}

\begin{abstract}
The physical nature of gamma-ray bursts (GRBs) are believed to involve an ultra-relativistic jet. The observed complex structure of light curves motivate the idea of jet precession. In this work, we study the gravitational waves of jet precession based on neutrino-dominated accretion disks around black holes, which may account for the central engine of GRBs. In our model, the jet and the inner part of the disk may precess along with the black hole, which is driven by the outer part of the disk. Gravitational waves are therefore expected to be significant from this black hole-inner disk precession system. By comparing our numerical results with the sensitivity of some detectors, we find that it is possible for DECIGO and BBO to detect such gravitational waves, particularly for GRBs in the Local Group.
\end{abstract}

Subject headings: accretion, accretion disks — black hole physics — gamma rays: bursts - gravitational waves

\section{Introduction}

Gamma-ray bursts (GRBs), which were first detected in 1967, are intense flashes of gamma rays followed by fainter multiple-wavelength (X-ray, optical, radio) emission named "afterglow". Physically, GRBs are believed to be emitted from ultra-relativistic jets. The afterglow emission is also expected as the jet interacts with the ambient medium (Piran 2004). One popular central engine model that powers GRBs consists of a black hole and a neutrinodominated accretion flow (NDAF) with mass accretion rates around $0.01 \sim 10 \mathrm{M}_{\odot} \mathrm{s}^{-1}$ (see, e.g., Popham et al. 1999; Narayan et al. 2001; Kohri et al. 2005; Lee et al. 2005; Gu et al.

\footnotetext{
${ }^{1}$ Department of Physics and Institute of Theoretical Physics and Astrophysics, Xiamen University, Xiamen, Fujian 361005, China

${ }^{2}$ Harvard-Smithsonian Center for Astrophysics, 60 Garden Street, Cambridge, MA 02138, USA
} 
2006; Chen \& Beloborodov 2007; Liu et al. 2007, 2008, 2012; Lei et al. 2009). Detailed studies have revealed that the quasi-periodic structure exists in a wide variety of observed light profiles of GRBs (Romero et al. 1999; Liang et al. 2004). One possible explanation (particularly for those with a fast rise and exponential decay, i.e.. "FRED") involves a precessing jet (e.g., Blackman et al. 1996; Portegies Zwart et al. 1999; Reynoso et al. 2006; Lei et al. 2007; Liu et al. 2010).

Blackman et al. (1996) investigated the precession of a relativistic blob-emitting neutron star jet in a binary pulsar (PSR-binary). They considered Newtonian tidal torque and gravitomagnetic interaction between PSR-binary to account for precession and nutation frequencies. Later, Portegies Zwart et al. (1999) studied a black hole-neutron star model for GRBs by the black hole forcing the accretion disk and the jet to precess due to the Newtonian tidal torque. Moreover, their study showed that the theoretical light curves can fit the observational data well if one considers the modulation of jet precession on intrinsic light curves. The gravitomagnetic interaction between the black hole and the accretion disk is, however, much stronger than the tidal force (Thorne et al. 1986). Reynoso et al. (2006) suggested the gravitomagnetic interaction as a new basis for jet precession in GRBs. The size of the accretion disk needs to be small enough to produce a precession period $\sim 0.5 \mathrm{~s}$, since in their model the whole disk precesses around the black hole. Furthermore, Lei et al. (2007) suggested that the modulated evolution of the half-opening angle may play the role, responsible for light curves of GRBs.

Another jet precession model in GRBs was proposed by Liu et al. (2010), which followed the previous scheme in X-ray binaries (e.g., Sarazin et al. 1980) and active galactic nuclei (e.g., Lu 1990; Lu \& Zhou 2005). Liu et al. (2010) argued that the outer neutrino-dominated accretion disk, whose angular momentum is sufficiently larger than that of the black hole, can maintain its orientation and force the black hole to precess. On the contrary, the inner disk whose angular momentum is significantly smaller than that of the black hole, should be aligned with the black hole spin axis (Bardeen \& Petterson 1975). Hence, the whole dynamical system becomes a precessing black hole-inner disk system. The ultra-relativistic jet, which is launched by the inner disk aligned with the black hole spin axis, will also precess and produce the observational complex structure in the light curves.

Apart from the electromagnetic emission in GRBs, gravitational waves are also expected due to the black hole-inner disk precession. Different from gamma-ray and afterglow emission, which is believed to be produced far from the black hole, the gravitational radiation should be emitted near the central engine. On the other hand, many efforts have been made to detect gravitational wave signals with some current detectors such as LIGO and some still undergoing detectors like DECIGO, BBO, and LISA. The main purpose of this work is to 
study the detectability of the above mentioned gravitational waves.

This paper is organized as follows. In Section 2 we describe the disk-driven jet precession model. In Section 3 we show the formulae for calculating gravitational waves emitted by the black hole-inner disk precession system. In Section 4 we present our numerical results and discuss the possibility of detecting such gravitational waves by some future detectors. Conclusions and discussion are made in Section 5.

\section{Disk-Driven Jet Precession Model}

In this section, we review the main features of the disk-driven jet precession model (for details, see Liu et al. 2010). The model we considered is a Kerr black hole surrounded by a tilted accretion disk whose initial orbital axis is misaligned with the black hole spinning axis. The angular momentum per each ring at radius $r$ with width $d r$ is $d J=2 \pi r^{2} \Sigma v_{\varphi} d r$, where $\Sigma$ is the surface density and $v_{\varphi}$ is the azimuthal velocity. A typical angular momentum of the disk is (e.g., Sarazin et al. 1980)

$$
J=\frac{d J}{d(\ln r)}=2 \pi r^{3} \Sigma v_{\varphi} .
$$

There exists a critical radius $r_{\mathrm{p}}$ where the typical angular momentum $\left.J\right|_{r=r_{\mathrm{p}}}$ is equal to the black hole angular momentum $J_{*}$, i.e.,

$$
\left.J\right|_{r=r_{\mathrm{p}}}=J_{*}=\frac{G M^{2} a_{*}}{c}
$$

where $M$ is the black hole mass and $a_{*}$ is a dimensionless spin parameter $\left(0<a_{*}<1\right)$. The outer disk $\left(r>r_{\mathrm{p}}\right)$ will maintain its orientation and therefore force the black hole and the inner disk $\left(r<r_{\mathrm{p}}\right)$ to be a whole precessing system. The precession rate is expressed as (e.g., Sarazin et al. 1980; Lu 1990)

$$
\Omega=\frac{2 G J_{*}}{c^{2} r_{\mathrm{p}}^{3}}
$$

The mass conservation equation takes the form,

$$
\dot{M}=-2 \pi r \Sigma v_{r}
$$

where $\dot{M}$ is the mass accretion rate and $v_{r}$ is the radial velocity of the flow. The precession period can be derived by combining Eqs. (1)-(4) to eliminate $\Sigma, r_{\mathrm{p}}$, and $J_{*}$,

$$
P=\frac{2 \pi}{\Omega}=\pi M\left(\frac{a_{*}}{G}\right)^{\frac{1}{2}}\left(-\frac{\left.c v_{r}\right|_{r=r_{\mathrm{p}}}}{\left.\dot{M} v_{\varphi}\right|_{r=r_{\mathrm{p}}}}\right)^{\frac{3}{2}} .
$$


Following Riffert \& Herold (1995), we introduce the general relativity correction as

$$
\begin{gathered}
A=1-\frac{2 G M}{c^{2} r}+\left(\frac{G M a_{*}}{c^{2} r}\right)^{2}, \\
B=1-\frac{3 G M}{c^{2} r}+2 a_{*}\left(\frac{G M}{c^{2} r}\right)^{3 / 2}, \\
C=1-4 a_{*}\left(\frac{G M}{c^{2} r}\right)^{\frac{3}{2}}+3\left(\frac{G M a_{*}}{c^{2} r}\right)^{2}, \\
D=\int_{r_{\mathrm{ms}}}^{r} \frac{x^{2} c^{4} /\left(2 G^{2}\right)-3 x M c^{2} / G+4\left(x a_{*}^{2} M^{3} c^{2} / G\right)^{1 / 2}-3 a_{*}^{2} M^{2} / 2}{(x r)^{1 / 2}\left[x^{2} c^{4} / G^{2}-3 x M c^{2} / G+2\left(x a_{*}^{2} M^{3} c^{2} / G\right)^{1 / 2}\right]} d x,
\end{gathered}
$$

where $r_{\mathrm{ms}}$ is the radius of inner marginally stable orbit. The hydrostatic balance in $z$ direction takes the form:

$$
\frac{1}{\rho} \frac{\partial p}{\partial z}=-\frac{G M z}{r^{3}} \frac{C}{B},
$$

where $\rho$ is the mass density, and $p$ is the total pressure. We make an improvement on describing the vertical structure by a polytropic relation, i.e., $p=K \rho^{1+1 / N}$, instead of the one-zone approximation in Liu et al. (2010), where $1+1 / N$ is the polytropic index. The above hydrostatic equation then gives (e.g., Hōshi 1977)

$$
\begin{gathered}
\rho(r, z)=\rho_{0}(r)\left(1-\frac{z^{2}}{H^{2}}\right)^{N}, \\
p(r, z)=p_{0}(r)\left(1-\frac{z^{2}}{H^{2}}\right)^{N+1},
\end{gathered}
$$

where $\rho_{0}$ and $p_{0}$ are the density and the pressure on the equatorial plane, respectively. The half-thickness of the disk, $H$, is expressed as

$$
\Omega_{\mathrm{K}}^{2} H^{2}=2(N+1) \frac{p_{0}}{\rho_{0}} \frac{B}{C},
$$

where $\Omega_{\mathrm{K}}$ is the Keplerian angular velocity. The above equations combined with the energy and momentum conservation equations and the equation of state (see, e.g., Liu et al. 2007) enable us to solve the structure of the disk and consequently to obtain the precession period. Then we can go further to investigate the property of gravitational waves from such a black hole-inner disk precession system.

\section{Gravitational waves from the black hole-inner disk precession system}

Here we consider the black hole-inner disk precessing system as an axisymmetric rigid system(with moments of inertia $I_{1}=I_{2}$ ). Such a system would precess with a period given 
in Equation (5). In the body frame, the inertia tensor is diagonal with eigenvalues $I_{1}, I_{2}, I_{3}$, where

$$
\begin{gathered}
I_{3}=\int_{r<r_{\mathrm{p}}}\left(x^{2}+y^{2}\right) \rho(x, y, z) d x d y d z, \\
I_{1}=I_{2}=\int_{r<r_{\mathrm{p}}}\left(z^{2}+y^{2}\right) \rho(x, y, z) d x d y d z .
\end{gathered}
$$

The precessing motion of such a rigid system is a classical Newtonian problem and it is easy to obtain the inertia tensor in the observing frame (e.g., Landau \& Lifshitz 1976; Maggiore 2008):

$$
\begin{gathered}
I_{x x}=\frac{1}{2}\left(I_{1}-I_{3}\right) \sin ^{2} \theta \cos (2 \Omega t)+C_{1}, \\
I_{x y}=\frac{1}{2} \sin ^{2} \theta \sin (2 \Omega t), \\
I_{y y}=-\frac{1}{2}\left(I_{1}-I_{3}\right) \sin ^{2} \theta \cos (2 \Omega t)+C_{2}, \\
I_{x z}=-\left(I_{1}-I_{3}\right) \sin \theta \cos \theta \sin (2 \Omega t), \\
I_{y z}=-I_{x z}, \\
I_{z z}=I_{1} \sin ^{2} \theta+I_{3} \cos ^{2} \theta,
\end{gathered}
$$

where $\theta$ is the misaligned angle between the black hole spin axis and the orientation of the outer disk, and $\Omega$ is the precession rate given by Equation (3). Note that $C_{1}$ and $C_{2}$ represent some constants which are unimportant in our calculation since gravitational waves are only relevant to the time-dependent components of moments of inertia.

Gravitational waves will be produced since the inertia tensor in the observing frame is time dependent. With the assumption that the angle between the $z$-axis of gravitational wave detector and the signal direction of arrival is $\iota$ and the distance of the GRB is $d$, the amplitude of gravitational waves is given by (e.g., Zimmermann \& Szedenits 1979; Maggiore 2008)

$$
h_{\text {pre }}(t)=h_{+}(t)+h_{\times}(t) \text {, }
$$

where

$$
\begin{gathered}
h_{+}(t)=h_{0} \sin 2 \theta \cos (\Omega t) \sin \iota \cos \iota \\
+2 h_{0} \sin ^{2} \theta \cos (2 \Omega t)\left(1+\cos ^{2} \iota\right), \\
h_{\times}(t)=h_{0} \sin 2 \theta \sin (\Omega t) \sin \iota \\
+4 h_{0} \sin ^{2} \theta \sin (2 \Omega t) \cos \iota,
\end{gathered}
$$


with

$$
h_{0}=-\frac{G}{c^{4}} \frac{\left(I_{3}-I_{1}\right) \Omega^{2}}{d} .
$$

Thus the black hole-inner disk system emits gravitational waves at two frequencies, i.e., $f_{\mathrm{gw}}=\Omega / 2 \pi$ and $\Omega / \pi$. Meanwhile, since the hyper-accretion in GRBs exists only for seconds and would finally stop, the gravitational wave is this work should be a gravitational wave burst and be significant only when the central engine is active. Thus, the duration of the gravitational wave burst is roughly equal to the activity time of the central engine of GRBs. The gravitational wave signal waveform is therefore expected to be (e.g., Maggiore 2008)

$$
h(t)=h_{\mathrm{pre}}(t) e^{-\frac{t^{2}}{2 \delta_{\mathrm{pla}}^{2}}}
$$

where $\delta_{\text {pla }}$ is the plateau time of GRBs, which is roughly the duration of activity time of the central engine. In order to assess the detectability, we calculate the root-sum-square (rss) amplitude as follows (e.g., Acernese et al. 2008; Maggiore 2008):

$$
h_{\mathrm{rss}}(f)=\sqrt{\int_{-\infty}^{\infty}\left(h_{+}^{2}(t)+h_{\times}^{2}(t)\right) d t},
$$

where $f=\Omega / 2 \pi=1 / P$.

Gravitational waves also carry energy and momentum. The quadrupole power of gravitational wave is

$$
P_{\text {quad }}=\frac{G}{5 c^{5}}\left\langle\dddot{M}_{i j} \dddot{M}_{i j}-\frac{1}{3}\left(\dddot{M}_{k k}\right)^{2}\right\rangle \text {. }
$$

In our case, the momentum $M_{i j}$ satisfies the relation $\dddot{M}_{i j}=\dddot{I}_{i j}$ (e.g., Maggiore 2008). Therefore, the quadrupole power radiated is

$$
P_{\text {quad }}=\frac{2 G}{5 c^{5}}\left(I_{1}-I_{3}\right)^{2} \Omega^{6} \sin ^{2} \theta\left(1+15 \sin ^{2} \theta\right) .
$$

\section{Numerical Results}

The structure of our jet precession model is determined by $\dot{M}, a_{*}, M$, and the viscosity parameter $\alpha$. Following Liu et al. (2010), we adopt $\alpha=0.01$ in our calculation. In addition, the polytropic index $1+1 / N$ is set to be $5 / 3$. We then numerically solve the equations described in Section 2 with given $\dot{M}, M$, and $a_{*}$ to obtain the structure of the system. In order to calculate the strength of gravitational waves, we assume that the misaligned angle between the black hole spinning axis and the accretion disk orientation is $\theta=20^{\circ}$. We also fix the duration gravitational wave bursts (roughly the activity time of the central engine 
of GRBs, which is, see e.g., Portegies Zwart et al. 1999, an order of $20 \mathrm{~s}) \delta_{\text {pla }}=20 \mathrm{~s}$ for illustration purpose. In addition, for given distance $d$ of the GRB source, we can derive the rss amplitude and quadrupole power of gravitational waves by equations in Section 3 .

\subsection{Disk-driven Jet Precession}

With fixed $M=6 \mathrm{M}_{\odot}$ and $\alpha=0.01$, we illustrate the possible jet precession period $P$ as a function of the accretion rate (solid line) in Figure 1, In addition, we plot the critical radius $r_{\mathrm{p}}$ as a function of the accretion rate (dashed line). It is seen that NDAFs with $\dot{M}=0.05 \sim 10 \mathrm{M}_{\odot} \mathrm{s}^{-1}$ can drive jet precession with period $P=10 \sim 0.1 \mathrm{~s}$. Thus the diskdriven jet precession may explain the temporal structures in light curves of GRBs. Note that the corresponding critical radius $r_{\mathrm{p}}$ is close to the horizon of the black hole and the inner precession disk is thus very small.

\subsection{Gravitational waves and the black hole spin}

It is known that the black hole spin can affect both the structure and the precession rate. In this section we study the dependence of the gravitational wave rss amplitude on the black hole spin for a fixed $M$ and $d$. We vary the spinning parameter from $a_{*}=0.1$ to $a_{*}=0.95$ and calculate the corresponding gravitational wave rss amplitude $h_{\mathrm{rss}}$.

Figure 2 plots the amplitude $h_{\mathrm{rss}}$ as a function of $a_{*}$, for which $M=6 \mathrm{M}_{\odot}$ and $d=1 \mathrm{Mpc}$. The solid, dashed, and dotted lines correspond to $\dot{M}=0.1,1$, and $10 \mathrm{M}_{\odot} \mathrm{s}^{-1}$, respectively. It is seen that $h_{\mathrm{rss}}$ rapidly increases with $a_{*}$ in the low-spin region, whereas $h_{\mathrm{rss}}$ becomes flat in the high-spin region. This result is easy to understand since the black hole with higher spin will precess along with a larger inner disk, and consequently the moments of inertia of the precession system will be larger, which will result in larger $h_{\mathrm{rss}}$.

The formation of an ultra-relativistic jet may require a rapid spinning black hole (e.g., Narayan \& McClintock 2012). The hyper accretion process would also spinning up the central black hole. Therefore, we fix $a_{*}=0.95$ for the later calculation to focus on the variation of $h_{\text {rss }}$ and frequency with varying $\dot{M}, M$, and $d$. 


\subsection{Gravitational waves and the precession rate}

For a given mass of the black hole, the disk structure and the precession period are determined only by $\dot{M}$ ( $\alpha$ and $a_{*}$ have been fixed), so there exists a certain $P$ (or $\left.f=1 / P\right)$ corresponding to each $\dot{M}$. In this case, if the distance is also given, then there also exists a certain $h_{\text {rss }}$ corresponding to each $\dot{M}$. In this section, we investigate the gravitational waves for each $\dot{M}$ (and $P, f)$.

As mentioned at the beginning of this section, we set $\theta=20^{\circ}$ to assess the rss amplitude

of gravitational waves since as shown by Revnoso et al. (2006), $\theta$ is an order of $20^{\circ}$ in some GRBs. However, $\theta$ may be different for different GRBs. In Figure 3 we present the rss amplitude of gravitational waves as a function of $f$ for $\theta=5^{\circ}$ (solid line) and $\theta=20^{\circ}$ (dashed line). It is seen that the rss amplitude decreases by a factor of 2 to 3 as $\theta$ varies from $20^{\circ}$ to $5^{\circ}$. We will keep $\theta=20^{\circ}$ for calculation in the remainder of this paper.

Figure 4 shows the relationship among $h_{\text {rss }}, f$, and $\dot{M}$, for which $M=6 \mathrm{M}_{\odot}$. The range of $\dot{M}$ is roughly $0.01 \sim 10 \mathrm{M}_{\odot} \mathrm{s}^{-1}$, which is known as the possible $\dot{M}$ for GRBs. The dash-dotted, solid, and dashed lines correspond to the results for $d=10 \mathrm{kpc}, 1 \mathrm{Mpc}$, and $100 \mathrm{Mpc}$, respectively. The dotted lines represent the detectability of DECIGO, BBO, and the ultimate DECIGO (e.g., Yagi \& Seto 2011). The figure indicates that, for GRBs in the Local Group $(d \lesssim 1 \mathrm{Mpc})$, such gravitational waves are under DECIGO and BBO's detectability. The event rate is, however, quite low in the Local Group. For larger distance $d \lesssim 100 \mathrm{Mpc}$, the event rate may increase to $\sim 1 \mathrm{yr}^{-1}$, but the rss amplitude $h_{\mathrm{rss}}$ is too small to be detected by practical DECIGO or BBO. Nevertheless, as shown in this figure, such gravitational waves are under detectability of the ultimate DECIGO, which roughly represents the quantum level noise of gravitational wave detectors.

It is also seen from Figure 4 that the amplitude $h_{\mathrm{rss}}$ and the frequency $f$ both increase with $\dot{M}$. This is because higher accretion rates generally correspond to higher mass density and therefore larger moments of inertia and larger gravitational wave rss amplitude. On the other hand, the critical radius $r_{\mathrm{p}}$, will become smaller for higher accretion rates, which results in higher precession rates.

We also study the strength of such gravitational waves for various central black hole masses. Figure 5 presents the variation of $h_{\text {rss }}$ with $f$ for a fixed distance $d=1 \mathrm{Mpc}$. The dashed, solid, and dash-dotted lines correspond to the black hole mass $M=3,6$, and $10 \mathrm{M}_{\odot}$, respectively. It is seen that $h_{\mathrm{rss}}$ increases with the black hole mass, which can be understood as follows. The critical radius $r_{\mathrm{p}}$ increases with the black hole angular momentum $J_{*}$ (or $M$ since $a_{*}$ is fixed), so the larger $M$ corresponds to the larger moments of inertia of the inner disk, and therefore stronger gravitational waves. We would like to point out that, even 
though larger black hole may produce stronger gravitational wave rss amplitude, $h_{\mathrm{rss}}$ cannot be essentially enhanced since the black hole mass in GRBs is limited, normally $M \lesssim 10 \mathrm{M}_{\odot}$.

\subsection{The power of gravitational wave}

In order to further explore the gravitational wave from our model, we calculate the quadrupole power of gravitational waves $P_{\text {quad }}$. Figure 6 plots $P_{\text {quad }}$ as a function of $f$, for which $M=6 \mathrm{M}_{\odot}$ and $a_{*}=0.95$. In addition, the luminosity range of GRBs is shown as

the shaded region (e.g., Zhang 2011). It is seen that the power of the gravitational wave is significantly less than the isotropic luminosity of the GRBs. The gravitational potential energy released in the accretion disk is mainly converted into the neutrino radiation rather than the gravitational radiation. Therefore, our assumption that the structure of NDAFs is not affected by the production of gravitational waves should be self-consistent.

\section{Conclusions and Discussion}

In this paper, we have studied the gravitational waves from GRBs with disk-driven jet precession. Based on the model in Liu et al. (2010), we have calculated gravitational wave rss amplitude and obtained the variation of the amplitude with the frequency for different values of $M$ and $d$. By comparing our numerical results with the sensitivity of some detectors, we have found that it is possible for DECIGO and BBO to detect such gravitational waves, particularly for GRBs in the Local Group.

The rate of GRBs in the Local Group is apparently low. Hence, the detection rate of gravitational waves by our model is quite low (see Leonor et al. 2009, for a detailed discussion on the gravitational wave event rate that associates with GRBs). However, such gravitational waves may still be detected from systems absence of GRB events. On one hand, there is strong evidence showing that X-Ray Flashes (XRFs) and GRBs are just two types of bursts with the same physical nature (e.g., Lamb et al. 2003). On the other hand, observational and theoretical arguments both indicate that the so-called "failed" GRBs exist if the jet is dirty (baryon-rich) and cannot breakthrough the envelope (e.g., Huang et al. 2002; Totani 2003). Meanwhile, we argue that disk-driven jet precession may be common in black hole accretion system since the only necessary condition is that the angular momentum of the initial accretion flow is misaligned with the black hole spinning axis. Thus the similar gravitational waves may also be produced both in "failed" GRBs and XRFs. Then the detection rate is probably related to the total rate of "failed" GRBs and XRFs and therefore 
increase significantly. For example, if the total rate approaches the SNe Ib/c event rate (of course this should just be regarded as an upper limit), the expected detection rate by our model will increase to $10^{-2} \sim 10^{-1} \mathrm{yr}^{-1}$ (for the $\mathrm{SNe} \mathrm{Ib} / \mathrm{c}$ rate, see, e.g., Podsiadlowski et al. 2004). More importantly, the gravitational wave signals may be the unique way to explore the nature of off-axis GRBs and "failed" GRBs except for the possible orphan afterglow emission. For example, the trigger time of the orphan afterglow can be measured if the above mentioned gravitational waves are detected. Then one can make a distinguish between off-axis GRBs and "failed" GRBs by studying the time evolution of the orphan afterglow (see details in Huang et al. 2002).

Some studies (e.g., Sago et al. 2004; Hiramatsu et al. 2005; Suwa \& Murase 2009) also focused on the low frequency gravitational waves (typically, $f \lesssim 10 \mathrm{~Hz}$ ) emitted from GRBs. Sago et al. (2004) studied the gravitational waves from the acceleration stage of GRB jets based on the internal shock model. The gravitational waves they studied have a "memory effect" (that is, gravitational waves would survive at the end of the jet acceleration stage) and do not depend on the energy form of jets (that is, whether the jet is powered by BZ process or neutrino annihilation). Hiramatsu et al. (2005) considered that the gravitational waves with "memory effect" from the neutrino-driven GRB jets. Such gravitational waves are expected to be stronger than that of Sago et al. (2004) since the neutrino luminosity is much higher than the energy released by matter in jets. Suwa \& Murase (2009), however, suggested gravitational waves with "memory effect" would generated because of anisotropic neutrino emission above the NDAF in GRBs. All these gravitational waves can be detected by the gravitational wave detectors such as LISA and DECIGO if the frequency is less than $1 \mathrm{~Hz}$ and the source is located at a few Mpc (note that for Suwa \& Murase 2009, gravitational waves whose frequencies $f \sim 100 \mathrm{~Hz}$ can also be detected by LIGO). There are many common features between our model and these studies. For example, gravitational waves are both generated near the central engine (especially for our model and that of Suwa \& Murase 2009), frequencies of gravitational waves are both very low and the detectable distances are both about several Mpc. One obvious difference is that in our model gravitational waves whose frequencies $f=1 \sim 10 \mathrm{~Hz}$ are more likely to be detected. Therefore, a simultaneously detection of all these type of gravitational waves may give us a new sight into the central engine of GRBs.

Other types of gravitational waves emitted from the central engine of GRBs have been studied by van Putten \& Levinson (2003) and Romero et al. (2010). The former suggested that an inner GRB engine consists of a Kerr black hole surrounded by a uniform magnetized torus, whose accretion is suspended because the black hole-torus interaction can transfer angular momentum to the torus or the disk and prevent the accreted materials from falling into the horizon. The instability develops in the black hole-torus system because magnetic fields 
break up the axisymetry and a large fraction of energy of this system is released by gravitational waves, which make GRBs to be the most powerful gravitational radiation sources. Obviously, the gravitational waves studied in this work are systematically lower than that of van Putten \& Levinson (2003) both on the amplitude and the frequency (the frequency of gravitational waves suggested by van Putten \& Levinson 2003, is a few hundred Hz). On the other hand, gravitational waves in van Putten \& Levinson (2003) that powered by the spinning black hole would slow down the spin and change the structure of the surrounding torus significantly. In this work, however, as Figure [6] shown, the power of gravitational waves is much lower than that of van Putten \& Levinson (2003). Gravitational waves in this work almost have no affect on the structure of NDAFs. We can study the structure of NDAFs and the production of gravitational waves separately.

Moreover, Romero et al. (2010) considered a new type of gravitational wave by assuming that the whole accretion disk precesses as a rigid body. They showed that gravitational waves from such a precessing system can be detected by advanced LIGO in the near future (e.g., Figure 5 in their paper). Since the calculation of gravitational wave emission in Romero et al. (2010) is relevant to the formulae developed for a torque-free precession system, the frequency in their model is significantly different from that in the present work. Furthermore, the rss amplitude of gravitational waves of Romero et al. (2010) should, in principle, decrease with increasing frequency since the size of the accretion disk decreases with increasing frequency (e.g., Figure 5 in their paper). On the contrary, the rss amplitude in our model increases with frequency as shown in Figures 4 and 5. Such a difference may help distinguish our model from theirs.

We thank the anonymous referee for very useful suggestions and comments. We thank Ye-Fei Yuan, Matias M. Reynoso, and Gustavo E. Romero for beneficial discussions. This work was supported by the National Basic Research Program (973 Program) of China under grant 2009CB824800, and the National Natural Science Foundation of China under grants 10833002,11073015 , and 11103015.

\section{REFERENCES}

Acernese, F., et al. 2008, Classical and Quantum Gravity, 25, 225001

Bardeen, J. M., \& Petterson, J. A. 1975, ApJ, 195, L65

Blackman, E. G., Yi, I., \& Field, G. B. 1996, ApJ, 473, L79

Chen, W.-X., \& Beloborodov, A. M. 2007, ApJ, 657, 383 
Gu, W.-M., Liu, T., \& Lu, J.-F. 2006, ApJ, 643, L87

Hiramatsu, T., Kotake, K., Kudoh, H., \& Taruya, A. 2005, MNRAS, 364, 1063

Hōshi, R. 1977, Prog. Theor. Phys., 58, 1191

Huang, Y. F., Dai, Z. G., \& Lu, T. 2002, MNRAS, 332, 735

Kohri, K., Narayan, R., \& Piran, T. 2005, ApJ, 629, 341

Lamb, D. Q., Donaghy, T. Q., \& Graziani, C. 2003, arXiv:astro-ph/0309463

Landau, L. \& Lifshitz, E. 1976, Mechanics (3rd ed.; Oxford: Butterworth-Heinmann)

Lee, W. H., Ramirez-Ruiz, E., \& Page, D. 2005, ApJ, 632, 421

Lei, W. H., Wang, D. X., Gong, B. P., \& Huang, C. Y. 2007, A\&A, 468, 563

Lei, W. H., Wang, D. X., Zhang, L., Gan, Z. M., Zou, Y. C., \& Xie, Y. 2009, ApJ, 700, 1970

Leonor, I., Sutton, P. J., Frey, R., Jones, G., Márka, S., \& Márka, Z. 2009, Classical and Quantum Gravity, 26, 204017

Liang, E. W., Dai, Z. G., \& Wu, X. F. 2004, ApJ, 606, L29

Liu, T., Gu, W.-M., Xue, L., \& Lu, J.-F. 2007, ApJ, 661, 1025

Liu, T., Gu, W.-M., Xue, L., \& Lu, J.-F. 2012, Ap\&SS, 337, 711

Liu, T., Gu, W.-M., Xue, L., Weng, S.-S., \& Lu, J.-F. 2008, ApJ, 676, 545

Liu, T., Liang, E.-W., Gu, W.-M., Zhao, X.-H., Dai, Z. G., \& Lu, J.-F. 2010, A\&A, 516, A16

Lu, J.-F. 1990, A\&A, 229, 424

Lu, J.-F., \& Zhou, B.-Y. 2005, ApJ, 635, L17

Maggiore, M., 2008, Gravitational Waves: Volume 1 Theory and Experiments (New York: Oxford Univ. Press)

Narayan, R., \& McClintock, J. E. 2012, MNRAS, 419, L69

Narayan, R., Piran, T., \& Kumar, P. 2001, ApJ, 557, 949

Piran, T. 2004, Reviews of Modern Physics, 76, 1143 
Podsiadlowski, P., Mazzali, P. A., Nomoto, K., Lazzati, D., \& Cappellaro, E. 2004, ApJ, 607, L17

Popham, R., Woosley, S. E., \& Fryer, C. 1999, ApJ, 518, 356

Portegies Zwart, S. F., Lee, C.-H., \& Lee, H. K. 1999, ApJ, 520, 666

Reynoso, M. M., Romero, G. E., \& Sampayo, O. A. 2006, A\&A, 454, 11

Riffert, H., \& Herold, H. 1995, ApJ, 450, 508

Romero, G. E., Reynoso, M. M., \& Christiansen, H. R. 2010, A\&A, 524, A4

Romero, G. E., Torres, D. F., Andruchow, I., Anchordoqui, L. A., \& Link, B. 1999, MNRAS, 308, 799

Sago, N., Ioka, K., Nakamura, T., \& Yamazaki, R. 2004, Phys. Rev. D, 70, 104012

Sarazin, C. L., Begelman, M. C., \& Hatchett, S. P. 1980, ApJ, 238, L129

Suwa, Y., \& Murase, K. 2009, Phys. Rev. D, 80, 123008

Thorne, K. S., Price, R. H., \& MacDonald, D. A. 1986, Black Holes: The Membrane Paradigm (New Haven: Yale Univ. Press)

Totani, T. 2003, ApJ, 598, 1151

van Putten, M. H. P. M., \& Levinson, A. 2003, ApJ, 584, 937

Yagi, K., \& Seto, N. 2011, Phys. Rev. D, 83, 044011

Zhang, B. 2011, Comptes Rendus Physique, 12, 206

Zimmermann, M., \& Szedenits, E., Jr. 1979, Phys. Rev. D, 20, 351 


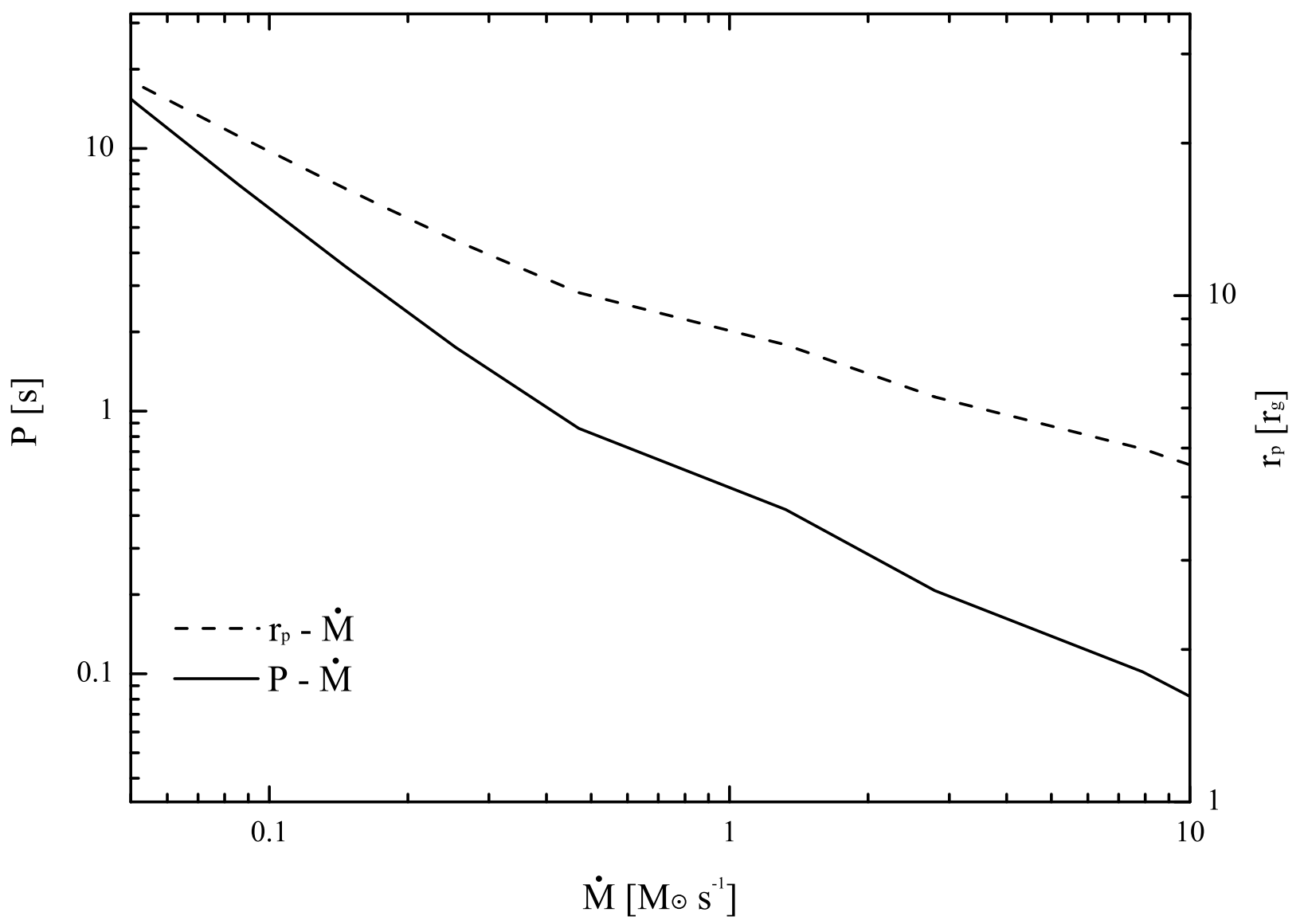

Fig. 1. - Variation of the precession period $P$ (solid line) and critical radius $r_{\mathrm{p}}$ (dashed line) with the accretion rate $\dot{M}$, for which $M=6 \mathrm{M}_{\odot}$ and $a_{*}=0.9$. 


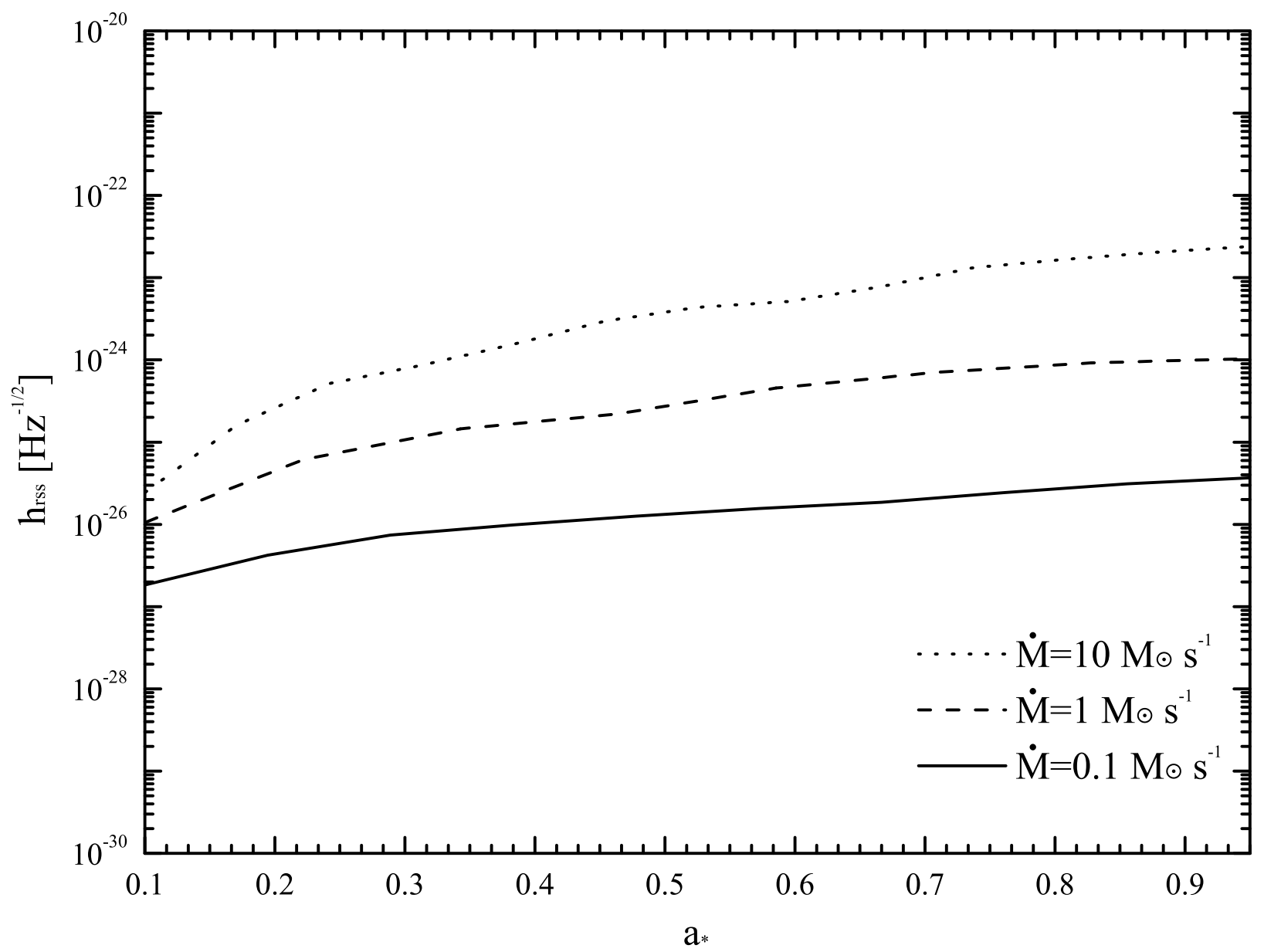

Fig. 2. - Variation of the gravitational wave rss amplitude with the spin parameter $a_{*}$, for which the black hole mass $M=6 \mathrm{M}_{\odot}$ and the distance $d=1 \mathrm{Mpc}$. The solid, dashed, and dotted lines correspond to the mass accretion rate $\dot{M}=0.1,1$, and $10 \mathrm{M}_{\odot} \mathrm{s}^{-1}$, respectively. 


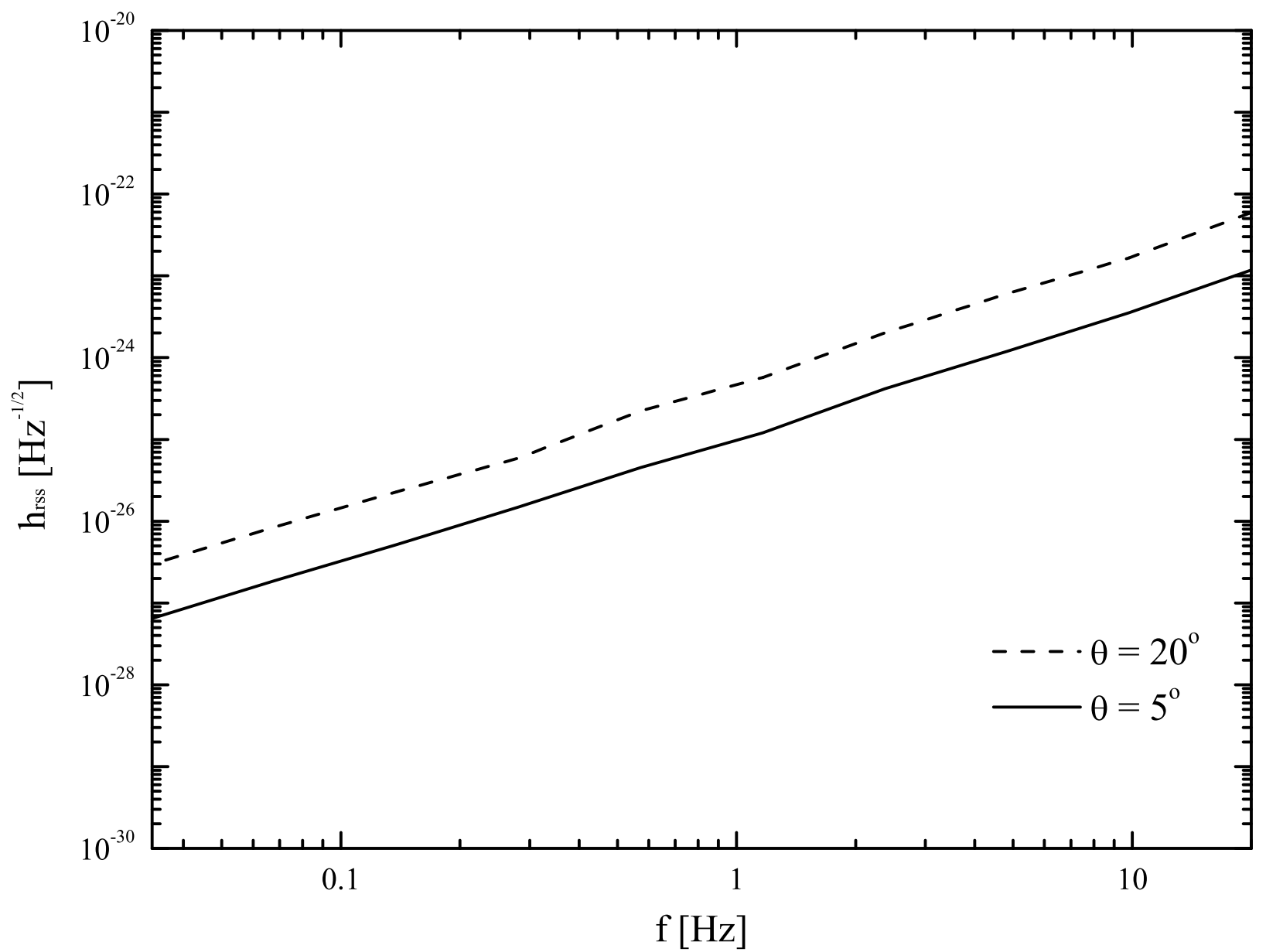

Fig. 3.- The gravitational wave rss amplitude as a function of the frequency for which $M=6 \mathrm{M}_{\odot}$ and $d=1 \mathrm{Mpc}$. The solid and dashed lines correspond to $\theta=5^{\circ}$ and $\theta=20^{\circ}$, respectively. 


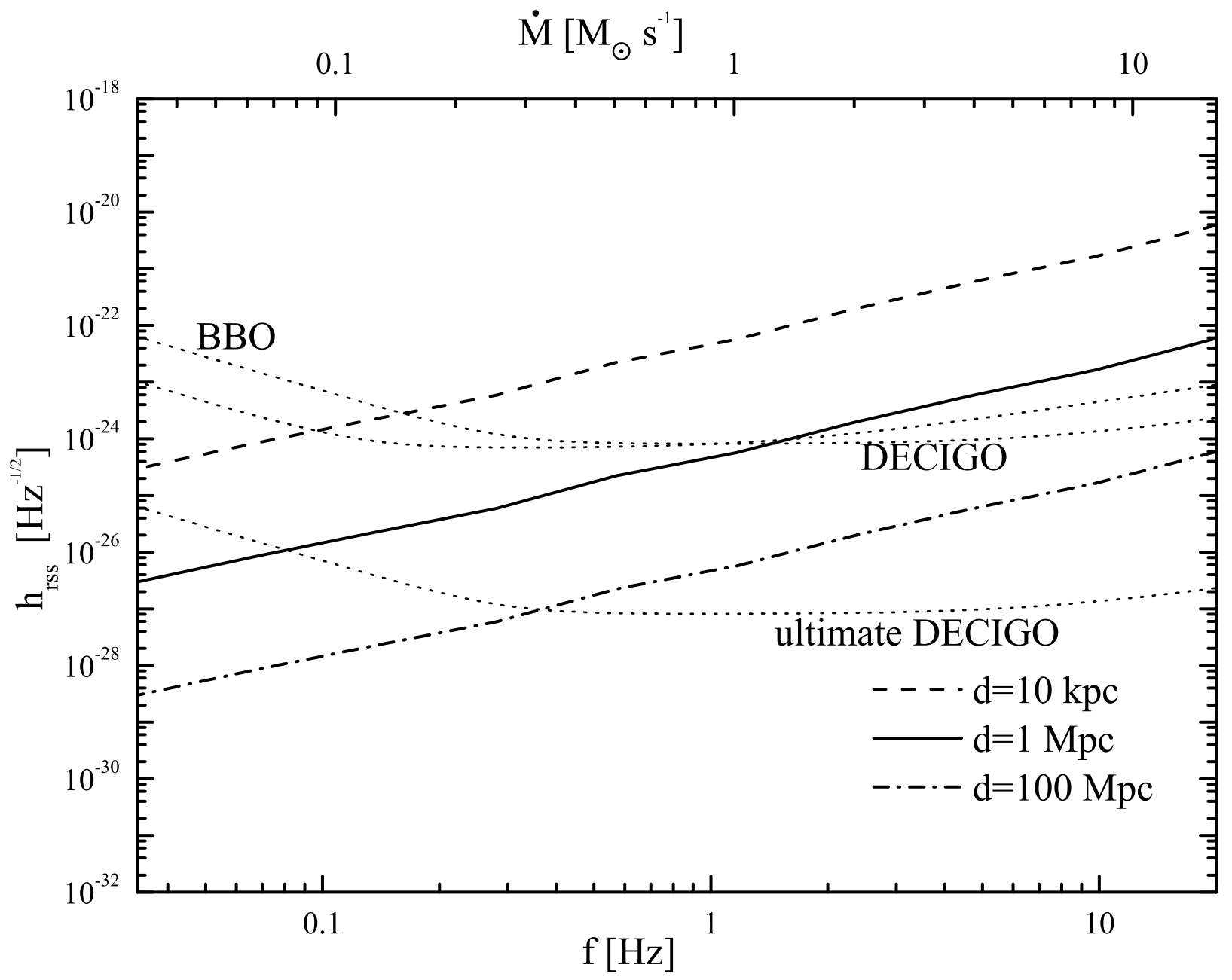

Fig. 4. - The gravitational wave rss amplitude as a function of the frequency (or the accretion rate), for which $M=6 \mathrm{M}_{\odot}$. The dashed, solid, and dash-dotted lines correspond to $d=$ $10 \mathrm{kpc}, 1 \mathrm{Mpc}$, and $100 \mathrm{Mpc}$, respectively. The dotted lines represent the detectability of gravitational wave detectors. 


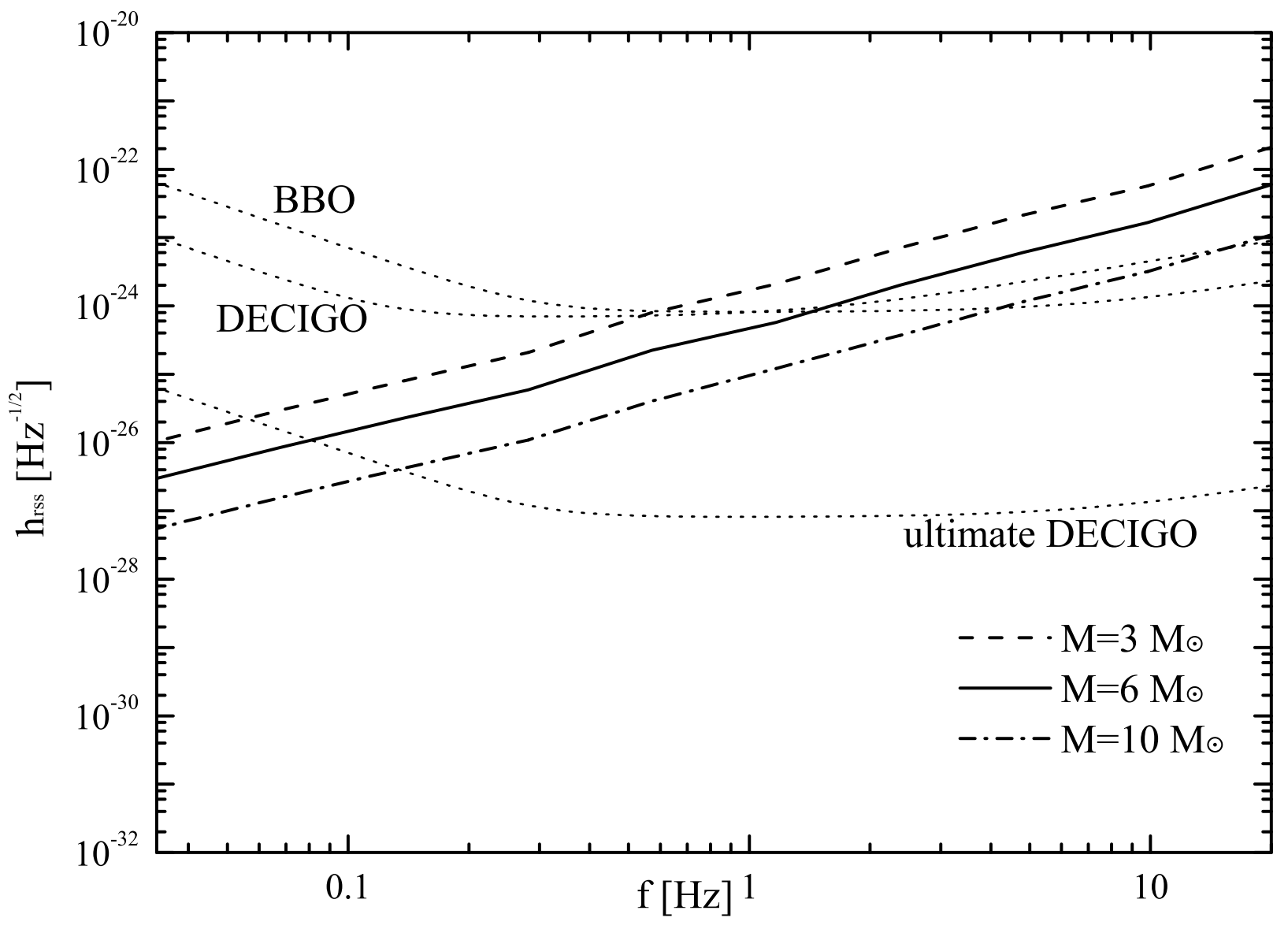

Fig. 5.- Same as Figure 2, except for $M=3,6$, and $10 \mathrm{M}_{\odot}$ with a fixed $d=1 \mathrm{Mpc}$. 


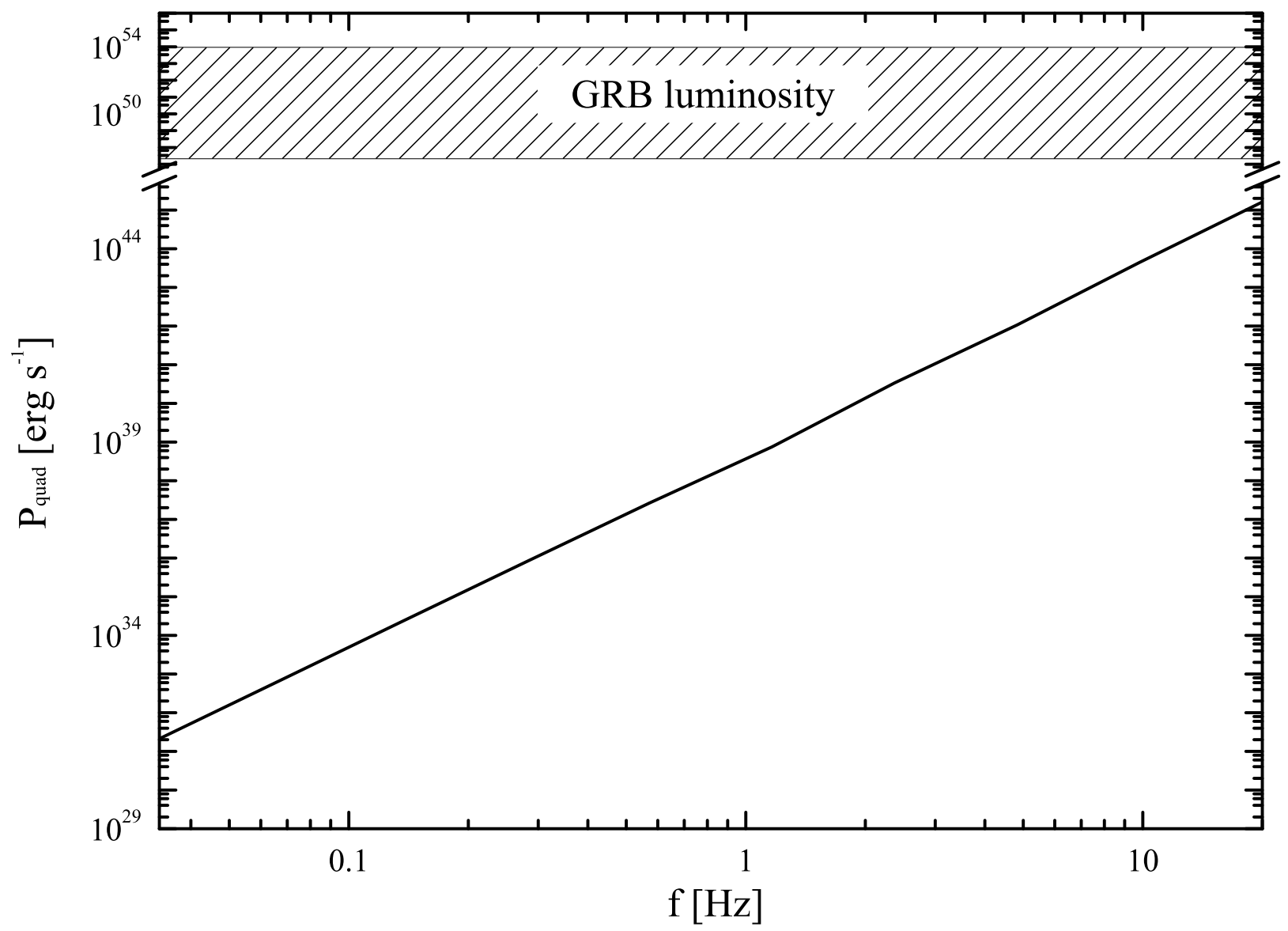

Fig. 6.- The quadruple power of gravitational wave radiated from the precessing central engine as a function of the frequency $f$, for which $M=6 \mathrm{M}_{\odot}$. The shaded region represents the luminosity range of GRBs. 